\title{
Micromotion Improvement and Applications for Abutment-Implant System by Uniform Design and Kriging Interpolation
}

\author{
Yung-Chang Cheng ${ }^{1 *}$, Deng-Huei Lin ${ }^{1}$, Cho-Pei Jiang² \\ ${ }^{1}$ Department of Mechanical and Automation Engineering, National Kaohsiung First University of Science \\ and Technology, Taiwan. \\ ${ }^{2}$ Department of Power Mechanical Engineering, National Formosa University, Taiwan. \\ * Corresponding author. Tel.: 0922988177; email: yccheng@nkfust.edu.tw \\ Manuscript submitted December 12, 2016; accepted April 6, 2016. \\ doi: 10.17706/ijbbb.2016.6.2.41-49
}

\begin{abstract}
This study aimed to analysis the micromotion of the3i, Branemark and Semados dental implant systems with Zimmer implant model subjected to dynamic chewing loads. Micromotion of the three dental implant systems with basic dimensions was obtained using dynamic finite element analysis. From the results, the best abutment type had been selected. Six parameters of the implant were selected as the control factors to be improved. A uniform design method was employed to construct a set of experimental simulations. Next, for each experimental simulation, the dynamic finite element analysis package ANSYS/LS-DYNA was employed to simulate the behavior of the Zimmer dental implant model subjected to dynamic chewing loads and then determined the maximum micromotion of the cortical and cancellous bones. Finally, the best design of the experimental simulations that caused the smallest amount of micromotion was selected as the improved design version. Compared to the original design, which experienced micromotion of $33.39 \mu \mu \mathrm{m}$, the improved version experienced micromotion of $22.22 \mu \mathrm{m}$. The rate of improvement was $33.45 \%$.Finally, the micromotion predicted system in commercial and engineering applications is constructed by Kriging interpolation method.
\end{abstract}

Key words: Zimmer implant model, abutment, dynamic chewing load, micromotion, uniform design, kriging interpolation.

\section{Introduction}

Dental implants are widely used as substitutes for missing teeth, to restore functionality and stability, and to improve upon the aesthetic appearance of a patient's mouth. A delay-load concept was employed to increase the stability to improve the success rate of dental implants. Typically, the healing period is several months. Instead of the delay-load concept, immediate loading was proposed to reduce the cost and time of implant treatment. Shafi et al. [1] investigated the stress transfer of various thread profiles and material properties of dental implant system by finite element analysis. Quaresma et al. [2] pre-sented the stress distribution of two types of dental implants with the abutment and implant under simulated occlusal forces. Using nonlinear finite element analysis method, Tang et al. [3] investigated the mechanical characteristics of three commonly used commercial dental implant systems with abutments. Kayabasi et al. [4] studied the dynamic behavior of dental implant systems with dynamic loadings. Neto et al. [5] illustrated the resonance 
frequency analysis of the abutment connection of dental implant systems. Ahmadian et al. [6] revealed the stress distribution of conventional tooth implants with a viscoelastic internal damping layer subjected to dynamic loadings. Many computational studies have attempted to simulate the loading effect on osseointegration using the finite element method, and most of these focus on implant interfaces under static loading and incorporate the shape design of the implant. However, this approach is only valid for static loading conditions, where there is no stress accumulation on the bone tissue.

In this study, 3D models of the 3i, Branemark and Semados dental implant systems which were comprised of the abutment, abutment screw, Zimmer implant, and cortical and cancellous bones. The micromotion of the three dental implant systems subjected to dynamic chewing loads was investigated by dynamic finite element analysis with basic dimensions. From the analysis results of the basic dimensions model, the dental implant system with the minimum micromotion is selected to investigate the improved design of the implant dimensions. Uniform design was employed to construct a set of experimental simulations. Finally, dimensions of an improved design for reducing the micromotion of the selected dental implant system subjected to dynamic chewing loads were presented.

\section{Analysis Method}

\subsection{Components of the Three Dental Implant Systems}

The geometric design of a Zimmer dental implant, is rebuilt using Pro/Engineer software and shown as Fig. 1. The some important parameters of the implant geometry are (A) screw pitch, (B) implant diameter, (C) screw depth, (D) maximum diameter of implant neck, (E) taper angle and (F) smaller diameter of implant neck, as listed in Table 1. In this article, 3i, Branemark and SemadosBranemark abutments are applied to comparing the micromotion. The three dental implant systems, 3i abutment in Fig. 2(a), Branemark abutment in Fig. 2(b), Semados in Fig. 2(c), including Zimmer implant, abutment, cortical and cancellous bones is shown as Fig. 2 [3].

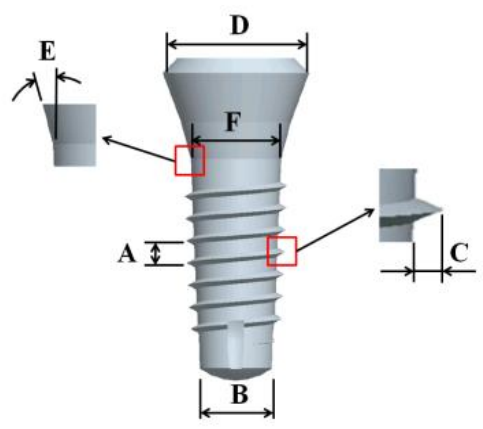

Fig. 1. 3D model of Zimmer implant.

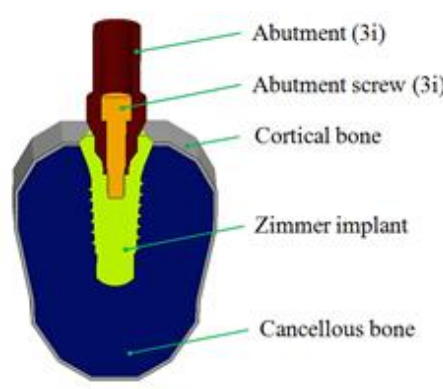

(a)

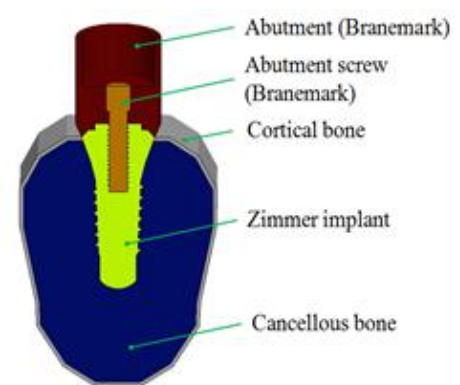

(b)

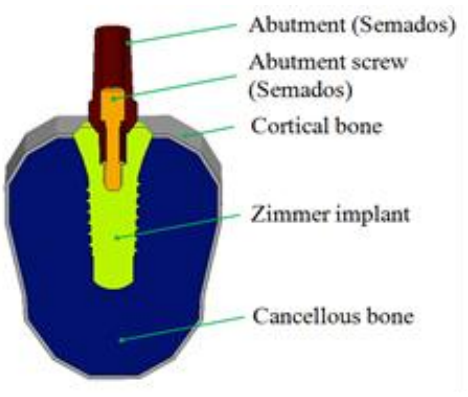

(c)

Fig. 2. Three dental implant systems with Zimmer implant [3]. 


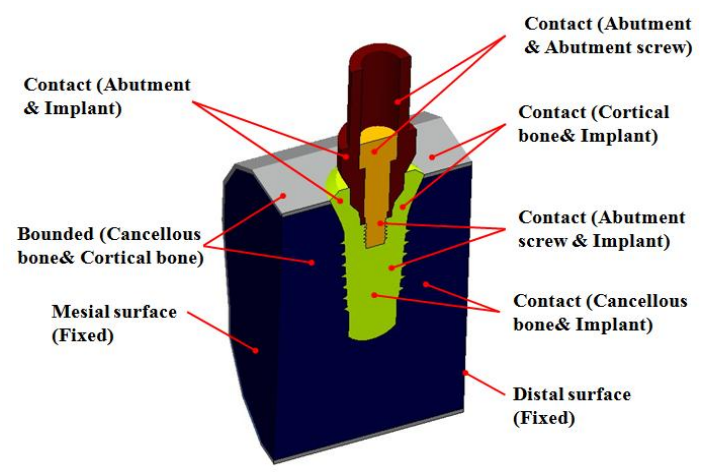

Fig. 3. Contact settings for finite element model.

Table 1. Geometric Properties of the Zimmer Implant

\begin{tabular}{cc}
\hline Item & Value \\
\hline Screw pitchA $(\mathrm{mm})$ & 0.9 \\
Body sizeB $(\mathrm{mm})$ & 3.0 \\
Screw depthC $(\mathrm{mm})$ & 0.33 \\
Maximum diameter of implant neckD $(\mathrm{mm})$ & 6.0 \\
Taper angleE $\left({ }^{\circ}\right)$ & 14.94 \\
Smaller diameter of implant neck F $(\mathrm{mm})$ & 4.02 \\
\hline
\end{tabular}
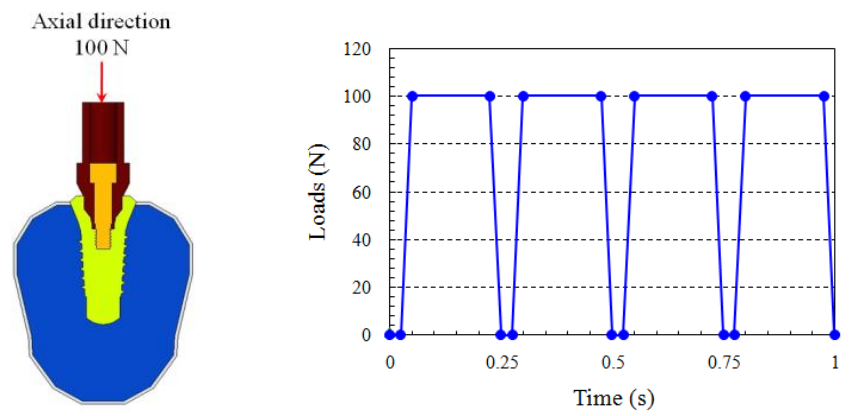

Fig. 4. Axial direction loading. Fig. 5. Dynamic loading components at fixed chewing rate.

\subsection{Dynamic Finite Element Analysis}

Preprocessor software, HyperMesh, was employed to mesh the models, which consisted of cortical bone, cancellous bone, an implant, and a contact region, due to the complicated geometry of each portion region, as shown in Fig. 3. The contact relationship between the implant and bone was defined to simulate the mechanical environment at the implant-bone interface following implant placement. The interfaces were assumed to be Coulomb frictional surfaces with a coefficient of 0.3 before osseointegration [7]. In addition, the boundary condition used for the assembled system was that the nodes on the mesial and distal bone surfaces were completely constrained in all directions; that is, nodal displacement components were set to zero.

In this study, the loading conditions were classified as dynamic. According to the literature (Chou et al. [7]), loads acting on the abutment are set as $100 \mathrm{~N}$ in the axial direction as shown in Fig. 4. By using dynamic finite element analysis, the time history of dynamic load components for a 1-second duration at a chewing rate of 4 cycles $(4 \mathrm{~Hz})$ per minute is shown in Fig. 5.

Micromotion ( $\mu$-motion) can occur in the region of the peri-implant during a loading and influences the primary implant stability. The degree of $\mu$-motion is also a key factor that determines whether an implantation is successful or not. After the mesh convergence analysis for each dental implant systems, the 
maximum value of micromotion of the 3i, Branemark and Semados dental implant systems are $62.47 \mu \mathrm{m}$, $35.32 \mu \mathrm{m}$ and $33.39 \mu \mathrm{m}$, and shown as in Figs. 6(a), 7(a) and 8(a). Figs 6(b), 7(b) and 8(b) are the dynamic responses of micromotion for three abutment-implant systems. It can be found that the micromotion is converged as time is increased. From the analysis results, the micromotion of the Semados dental implant system is the minimum. Therefore, the Semados dental implant system is chosen to study the improved design of the dimensions of the Zimmer implant model.

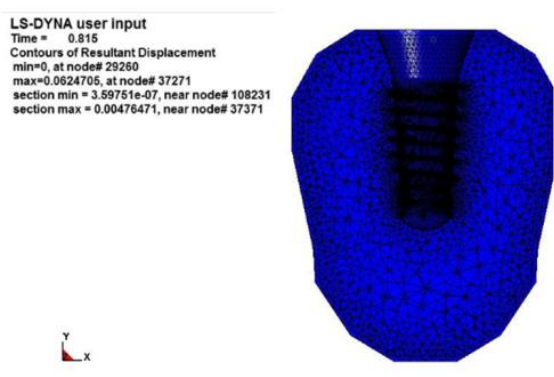

(a)

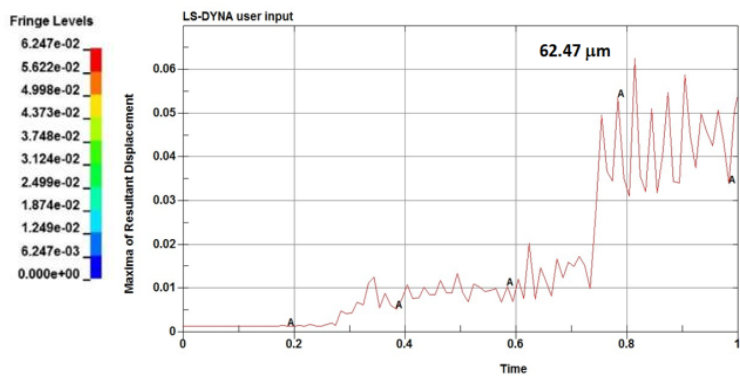

(b)

Fig. 6. (a) Contours (a) and dynamic response of micromotion (b) of dental implant system with $3 \mathrm{i}$ abutment.

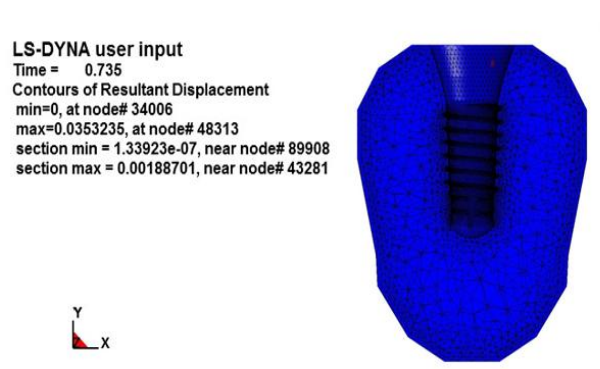

(a)

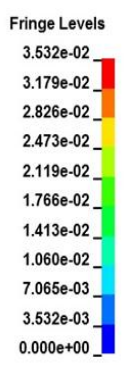

$0.000 \mathrm{e}+00]$

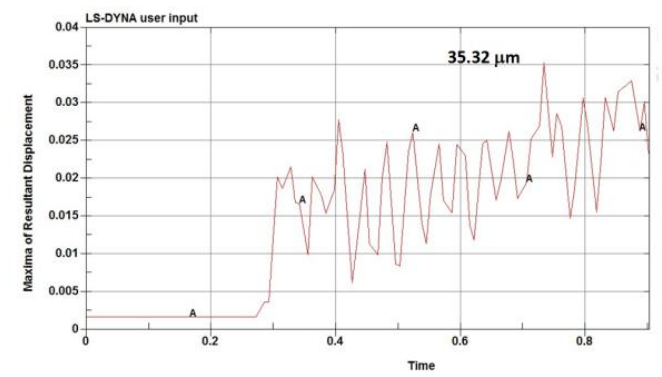

(b)

Fig. 7. (a) Contours (a) and dynamic response of micromotion (b) of dental implant system with Branemark abutment.

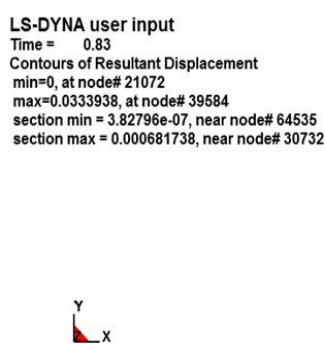

(a)
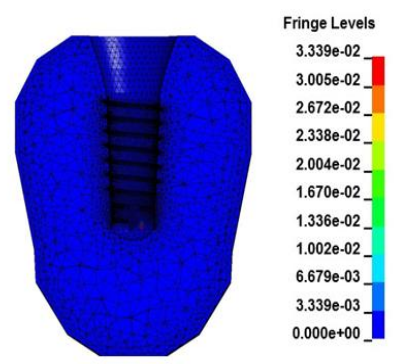

$0.000 \mathrm{e}+00]$

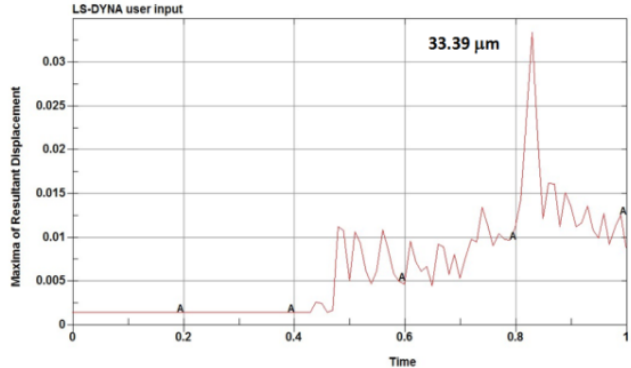

(b)

Fig. 8. (a) Contours (a) and dynamic response of micromotion (b) of dental implant system with Semados abutment.

\section{Micromotion Reduction and Results}

As shown in Fig. 1, the Zimmer implant model used in this study had four dimensional control factors: screw pitch (SP), screw depth (SD), body size (BS), and taper angle (TA). Two material control factors were 
the density of cancellous bone (Den) and Young's modulus of cancellous bone (EC). The original design and variation ranges of the control factors are shown in Table 3. Since all factors were continuous, the design space was also a continuous space. For a continuous design space, the design points are infinite and evaluation of all design points is impossible. Therefore, this study applied the uniform design of experiments, proposed by Fang and Wang [8], to construct a set of sample points which were scattered uniformly in the continuous design space. In this study, owing to the limitation of computational resources, each factor was divided into 16 levels and the uniform table $U_{16}^{*}\left(16^{12}\right)$ was utilized to construct 16 experiments asshown in Table 4. Since the Semados dental implant system had six control factors, columns $1,3,5,8,10$ and 11 were used according to the uniform table. The constructed experiments are shown in Table 5. Each experiment denoted a specific design of Semados dental implant system. For each Semados dental implant system, Pro/Engineer was employed to build the geometric model of frame, and ANSYS/LS-DYNA was utilized to simulate the behavior of the Semados dental implant system subjected to dynamic chewing loads [9]. Since the minimum micromotion occurred in the ninth experiment, the Semados dental implant system in the ninth experiment was regarded as the improved version of the design. The original design of the Semados dental implant system experienced micromotion of $33.39 \mu \mathrm{m}$. The improved version experienced micromotion of $22.22 \mu \mathrm{m}$, indicating a successful reduction in micromotion of the dental implant system, obtained with an increase in screw pitch, and decrease in body size and screw depth.

Table 2. Materials Properties Used in the Finite Element Model [7]

\begin{tabular}{cccc}
\hline Material & Young's modulus E(GPa) & Poisson's ratio & Density $\left(\mathrm{mg} / \mathrm{cm}^{3}\right)$ \\
\hline Implant & 110 & 0.35 & 4500 \\
Cortical bone & 13 & 0.3 & 2400 \\
Cancellous bone & 0.345 & 0.3 & 1100 \\
Abutment & 110 & 0.35 & 4500 \\
Abutment screw & 110 & 0.35 & 4500 \\
\hline
\end{tabular}

Table 3. Design Ranges of Control Factors

\begin{tabular}{ccccc}
\hline Control factor & Notation & Lower bound & Basic value & Upper bound \\
\hline Screw Pitch $(\mathrm{mm})$ & SP & 0.7 & 0.9 & 1.1 \\
Body Size $(\mathrm{mm})$ & BS & 2.3 & 3.0 & 3.5 \\
Screw Depth $(\mathrm{mm})$ & SD & 0.3 & 0.33 & 0.5 \\
Taper Angle $\left(^{\circ}\right)$ & TA & 10.0 & 14.94 & 18.0 \\
Density $\left(\mathrm{mg} / \mathrm{cm}^{3}\right)$ & Den & 960 & 1100 & 1200 \\
Young's modulus(MPa) & EC & 200 & 345 & 650 \\
\hline
\end{tabular}

\section{Micromotion Predicted System}

The surrogate modeling method adopted herein is Kriging Interpolation. Kriging, named after the South African mining engineer D.C. Krige, is a geo-statistical interpolation technique that considers both the distanceand the degree of variation between known data points when estimating values in unknown area[10].Kriging interpolation has been applied to studythe optimization problems in engineering [11]-[13].

To achieve the application for dentistry clinical medicine and medicine engineering, the micromotion 
predicted system is built and constructed by MATLABKriging toolbox. Applying the Kriging surrogate model of micromotion, the operation interface of the micromotion predicted system is obtained and given as shown in Fig. 9. The values of six control factors are input by user and press the calculating bottom. Then, the predicted micromotion is obtained. It is very convenience for dentist to predict the micromotion of the selected implant as the density and Yung's modulus of cancellous bone of patients are estimated and obtained.

Table 4. Uniform Table $U_{16}^{*}\left(16^{12}\right)$

\begin{tabular}{ccccccccccccc}
\hline No. & 1 & 2 & 3 & 4 & 5 & 6 & 7 & 8 & 9 & 10 & 11 & 12 \\
\hline 1 & 1 & 2 & 4 & 5 & 6 & 8 & 9 & 10 & 13 & 14 & 15 & 16 \\
2 & 2 & 4 & 8 & 10 & 12 & 16 & 1 & 3 & 9 & 11 & 13 & 15 \\
3 & 3 & 6 & 12 & 15 & 1 & 7 & 10 & 13 & 5 & 8 & 11 & 14 \\
4 & 4 & 8 & 16 & 3 & 7 & 15 & 2 & 6 & 1 & 5 & 9 & 13 \\
5 & 5 & 10 & 3 & 8 & 13 & 6 & 11 & 16 & 14 & 2 & 7 & 12 \\
6 & 6 & 12 & 7 & 13 & 2 & 14 & 3 & 9 & 10 & 16 & 5 & 11 \\
7 & 7 & 14 & 11 & 1 & 8 & 5 & 12 & 2 & 6 & 13 & 3 & 10 \\
8 & 8 & 16 & 15 & 6 & 14 & 13 & 4 & 12 & 2 & 10 & 1 & 9 \\
9 & 9 & 1 & 2 & 11 & 3 & 4 & 13 & 5 & 15 & 7 & 16 & 8 \\
10 & 10 & 3 & 6 & 16 & 9 & 12 & 5 & 15 & 11 & 4 & 14 & 7 \\
11 & 11 & 5 & 10 & 4 & 15 & 3 & 14 & 8 & 7 & 1 & 12 & 6 \\
12 & 12 & 7 & 14 & 9 & 4 & 11 & 6 & 1 & 3 & 15 & 10 & 5 \\
13 & 13 & 9 & 1 & 14 & 10 & 2 & 15 & 11 & 16 & 12 & 8 & 4 \\
14 & 14 & 11 & 5 & 2 & 16 & 10 & 7 & 4 & 12 & 9 & 6 & 3 \\
15 & 15 & 13 & 9 & 7 & 5 & 1 & 16 & 14 & 8 & 6 & 4 & 2 \\
16 & 16 & 15 & 13 & 12 & 11 & 9 & 8 & 7 & 4 & 3 & 2 & 1 \\
\hline & & & & & & & & & &
\end{tabular}

Table 5. Constructed Experiments and Results

\begin{tabular}{cccccccc}
\hline No. & $\mathrm{SP}(\mathrm{mm})$ & $\operatorname{Den}\left(\mathrm{mg} / \mathrm{cm}^{3}\right)$ & $\mathrm{SD}(\mathrm{mm})$ & $\mathrm{BS}(\mathrm{mm})$ & $\mathrm{TA}\left({ }^{\circ}\right)$ & $\mathrm{EC}(\mathrm{MPa})$ & Micromotion $(\mu \mathrm{m})$ \\
\hline 1 & 0.70 & 1008 & 0.37 & 3.02 & 16.93 & 620 & 54.79 \\
2 & 0.73 & 1072 & 0.45 & 2.46 & 15.33 & 560 & 48.61 \\
3 & 0.75 & 1136 & 0.30 & 3.26 & 13.73 & 500 & 33.93 \\
4 & 0.78 & 1200 & 0.38 & 2.70 & 12.13 & 440 & 33.87 \\
5 & 0.81 & 992 & 0.46 & 3.50 & 10.53 & 380 & 36.88 \\
6 & 0.83 & 1056 & 0.31 & 2.94 & 18.00 & 320 & 54.85 \\
7 & 0.86 & 1120 & 0.39 & 2.38 & 16.40 & 260 & 50.90 \\
8 & 0.89 & 1184 & 0.47 & 3.18 & 14.80 & 200 & 29.87 \\
9 & 0.91 & 976 & 0.33 & 2.62 & 13.20 & 650 & 22.22 \\
10 & 0.94 & 1040 & 0.41 & 3.42 & 11.60 & 590 & 41.27 \\
11 & 0.97 & 1104 & 0.49 & 2.86 & 10.00 & 530 & 40.08 \\
12 & 0.99 & 1168 & 0.34 & 2.30 & 17.47 & 470 & 39.60 \\
13 & 1.02 & 960 & 0.42 & 3.10 & 15.87 & 410 & 30.74 \\
14 & 1.05 & 1024 & 0.50 & 2.54 & 14.27 & 350 & 51.72 \\
15 & 1.07 & 1088 & 0.35 & 3.34 & 12.67 & 290 & 33.94 \\
16 & 1.10 & 1152 & 0.43 & 2.78 & 11.07 & 230 & 51.89 \\
\hline
\end{tabular}




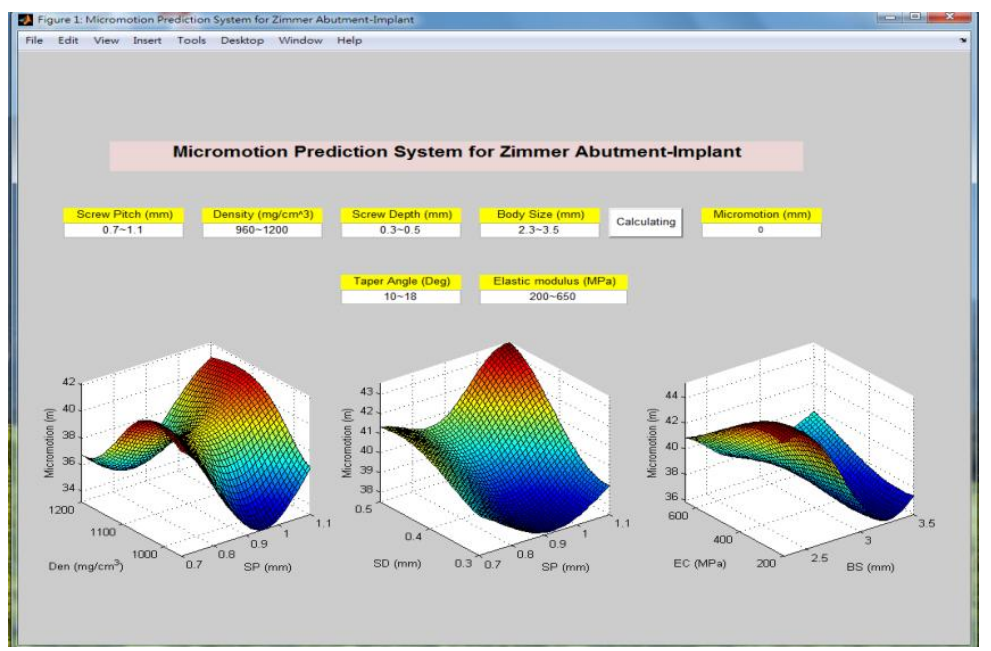

Fig. 9. Micromotion predicted system interface.

\section{Conclusion}

The dynamic finite element analysis of the 3i, Branemark and Semados dental implant systems with the Zimmer implant model has been investigated under the dynamic chewing loads. From the results, Semados dental implant system is selected to study the improved design of the dimensions of the Zimmer implant model because the micromotion of the Semados dental implant system is the smallest of the three dental implant systems. Besides that, this paper has completed the improvement of micromotion for the Semados dental implant system subjected dynamic chewing loads by using uniform design method and dynamic finite element analysis. For the original design, the micromotion is $33.39 \mu \mathrm{m}$. After executing uniform design improvement, the micromotion will go down to $22.22 \mu \mathrm{m}$. The rate of improvement is $33.45 \%$. This paper has shown that uniform design is a useful tool to reduce the micromotion for the Semados dental implant system with the Zimmer implant model subjected dynamic chewing loads. Finally, the micromotion predicted system in commercial and engineering applications is built and presented by Kriging interpolation method. These finding have not modeled the fracture of bone, the effect of blood flow and remodeling. Concerning such aspects into the simulation increases the complexity of the problem and modeling. Therefore, limitations of this study are simplified homogeneous and isotropic material properties used.

\section{Acknowledgment}

The authors acknowledge gratefully the financial support provided to this study by the Ministry of Science and Technology of Taiwan under grant numbers 104-2221-E-327-019, 104-2221-E-150 -030, 104-2218-E-010 -004 and Southern Taiwan Science Park under grant numbers 104CB04.

\section{References}

[1] Chen, S., Mulgrew, B., \& Grant, P. M. (2013). The effect of dental implant materials and thread profiles A finite element and statistical study. Journal of Medical Imaging and Health Informatics, 3(4), 509-513.

[2] Quaresma, S. E., Cury, P. R., Sendyk, W. R., \& Sendyk, C. (2008). A finite element analysis of two different dental implants: stress distribution in the prosthesis, abutment, implant, and supporting bone. Journal of Oral Implantology, 34(1), 509-513。

[3] Tang, C. B., Liu, S. Y., Zhou, G. X., Yu, J. H., Zhang, G. D., Bao, Y. D., \& Wang, Q. J. (2012). Nonlinear finite element analysis of three implant-abutment interface designs. International Journal of Oral Science, 
4(2), 101-108.

[4] Kayabasi, O., Yüzbasioglu, E., \& Erzincanli, F. (2006). Static, dynamic and fatigue behaviors of dental implant using finite element method. Journal of Advances in Engineering Software, 37(10), 649-658.

[5] Neto, M. A., Nicolau, P., Rocha, S., \& Leal, R. P. (2012). Finite element comparison of dental implants: Static and dynamic analysis. Proceedings of the 8th International Conference on Engineering Computational Technology, Dubrovnik, Croatia (pp. 1898-1909).

[6] Ahmadian, M. T., Firoozbakhsh, K., \& Ghanati, G. (2010). The dynamic analysis of a novel dental implant with a viscoelastic internal damping layer. Proceedings of the 17th Iranian Conference of Biomedical Engineering (pp. 1-4). Isfaham, Iran.

[7] Chou, I. C., Lee, S. Y., Wu, M. C., Sun, C. W., \& Jiang, C. P. (2014). Finite element modelling of implant designs and cortical bone thickness on stress distribution in maxillary type IV bone. Journal of Computer Methods in Biomechanics and Biomedical Engineering, 17(5), 516-526.

[8] Fang, K. T., \& Wang, Y. (1994). Number-Theoretic Methods in Statistics, Chapman \& Hall. London, U.K.

[9] Su, K. C., Chang, C. H., Chuang, S. F., \& Ng, E. Y. K. (2013). Biomechanical evaluation of endodontic post-restored teeth - Finite element analysis. Journal of Mechanics in Medicine and Biology, 13(1), 1350012-1-1350012-15.

[10] Lophaven, S. N., Nielsen, H. B., \& Søndergaard, J. (2002). DACE - A MATLAB krigingtoolbox. Technical Report of Informatics and Mathematical Modelling.

[11] Gu, Y. T., Wang, Q. X., \& Lam, K. Y. (2007). A meshless local kriging method for large deformation analyses. Computer Methods in Applied Mechanics and Engineering, 196(9-12), 1673-1684.

[12] McLean, P., Léger, P., \& Tinawi, R. (2006). Post-processing of finite element stress fields using dual kriging based methods for structural analysis of concrete dams. Finite Elements in Analysis and Design, 42(6), 532-546.

[13] Simpson, T. W., Mauery, T. M., Korte, J. J., \& Mistree, F. (2001). Kriging models for global approximation in simulation-based multidisciplinary design optimization. AIAA Journal, 39(12), 2233-2241.
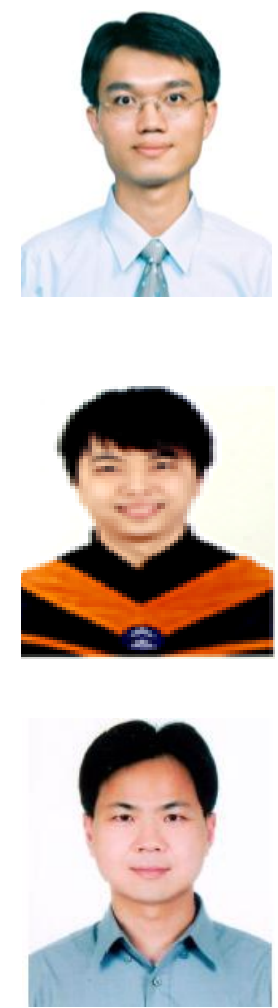

Yung-Chang Cheng is associate professor, the Department of Mechanical and Automation Engineering, National Kaohsiung First University of Science and Technology, Kaohsiung, Taiwan. He received his PhD from the Department of Mechanical Engineering,National Cheng Kung University, Tainan, Taiwan. His research interests include railway vehicle dynamics, vibration analysis, dynamic finite element analysis, optimization design, dental implant design and innovative engineering.

Deng-Huei Lin is graduated student, the Department of Mechanical and Automation Engineering, National Kaohsiung First University of Science and Technology, Kaohsiung, Taiwan, R.O.C.. He received his master degree from the Department of Mechanical and Automation Engineering, National Kaohsiung First University of Science and Technology. His research interests optimization design, dental implant analysis and design.

Cho-Pei Jiang is an associate professor in the Department of Power Mechanical Engineering of National Formosa University. He is the member of several famous association/societies including Taiwan Society for Technology of Plasticity (TSTP), Additive Manufacturing Association of Taiwan (AMAT) and International Institution for Micro Manufacturing (I2M2). He is also the executive secretary of TSTP and AEPA2014. Dr. Jiang has act as the reviewer for many academic journals, such as Rapid Prototyping 
Journal (RPJ), International Journal of Advanced Manufacturing Technology (IJAMT), Journal of Biomechanics (JBM), International Journal for Numerical Methods in Biomedical Engineering (IJNMBE) and International Journal of Precision Engineering and Manufacturing (IJPEM). His research areas are in the development and medical application of three-dimensional printing (3DP), metallic micro-forming, computer-aided design of dental restoration and dental materials. 